\title{
ARTICLE
}

Received 4 Dec 2015 | Accepted 22 Jun 2016 | Published 2 Aug 2016

DOl: $10.1038 /$ ncomms12309

OPEN

\section{A critical role for NF2 and the Hippo pathway in branching morphogenesis}

Antoine Reginensi ${ }^{1}$, Leonie Enderle ${ }^{1}$, Alex Gregorieff ${ }^{1}$, Randy L. Johnson ${ }^{2}$, Jeffrey L. Wrana ${ }^{1,3}$ \& Helen McNeill,3

Branching morphogenesis is a complex biological process common to the development of most epithelial organs. Here we demonstrate that NF2, LATS1/2 and YAP play a critical role in branching morphogenesis in the mouse kidney. Removal of $N f 2$ or Lats $1 / 2$ from the ureteric bud (UB) lineage causes loss of branching morphogenesis that is rescued by loss of one copy of Yap and Taz, and phenocopied by YAP overexpression. Mosaic analysis demonstrates that cells with high YAP expression have reduced contribution to UB tips, similar to Ret $^{-/}{ }^{-}$cells, and that YAP suppresses RET signalling and tip identity. Conversely, Yap/Taz UB-deletion leads to cyst-like branching and expansion of UB tip markers, suggesting a shift towards tip cell identity. Based on these data we propose that NF2 and the Hippo pathway locally repress YAP/TAZ activity in the UB to promote subsequent splitting of the tip to allow branching morphogenesis.

\footnotetext{
${ }^{1}$ Lunenfeld-Tanenbaum Research Institute, Mount Sinai Hospital, Toronto, Ontario, Canada M5G 1X5. ${ }^{2}$ Department of Biochemistry \& Molecular Biology, University of Texas MD Anderson Cancer Center, Houston, Texas 77030, USA. ${ }^{3}$ Department of Molecular Genetics, University of Toronto, Toronto, Ontario, Canada M5G 1X5. Correspondence and requests for materials should be addressed to A.R. (email: reginensi@lunenfeld.ca) or to H.M. (email: mcneill@lunenfeld.ca).
} 
B ranching morphogenesis is critical for the development and function of most epithelial organs, and is essential for the formation of the mammalian kidney. The kidney develops through reciprocal signalling between a ureteric epithelium that forms the collecting duct, and surrounding mesenchymal nephron progenitors and stroma ${ }^{1}$. The bilateral symmetry and characteristic shape of kidneys indicates branching morphogenesis is highly controlled. In addition, the rotational angle between one set of branches and the next is relatively fixed implying tight regulation ${ }^{2}$. How this developmentally critical branching is so tightly regulated is still unclear.

The first step of kidney development occurs when the ureteric bud (UB) invades the metanephric mesenchyme. Tip identity is defined in response to metanephric mesenchyme-derived signals including Glial cell-line-derived neurotrophic factor (GDNF) and fibroblast growth factors ${ }^{3}$. The tip contains progenitor cells that can self-renew or be left behind to give rise to trunk cells ${ }^{4}$. Binding of GDNF to its receptors GFR $\alpha 1$ and RET, triggers tyrosine kinase signalling, which induces the outgrowth of the UB. Once the ureter has invaded the metanephric mesenchyme, GDNF/RET signalling at UB tips leads to growth and repetitive branching of the ureter to form a ureteric tree that will give rise to the collecting duct. Loss of Gdnf, Ret or Gfral impairs branching morphogenesis causing kidney defects ranging from renal dysplasia to complete agenesis ${ }^{5-7}$. RET signalling is essential to form and maintain tips, and promotes a feed-forward signalling loop, in which RET signalling promotes expression of Ret transcript. The tip domain swells to form an ampulla before a symmetry breaking event occurs that allows it to split into two new tips. How symmetry is broken in the ampulla to form a branch is not understood.

Neurofibromatosis 2 ( $N f 2$ ) encodes a large FERM (4.1 protein/ ezrin/radixin/moesin)-domain containing protein also known as MERLIN. Mutations in NF2 are responsible for Neurofibromatosis type 2, a dominantly inherited tumour predisposition syndrome, characterized by the formation of benign neural tumours $^{8-10}$. Despite extensive research, the mechanisms by which mutations in NF2 cause disease remain unclear, due in part to multiple roles of NF2 in controlling several signalling pathways including PI3K-AKT, RAC-PAK, FAK-SRC and EGFR-RAS-ERK (ref. 10).

Studies both in flies and mammals suggest that NF2 can also regulate the Hippo pathway. The Hippo pathway is a conserved kinase cassette that regulates tissue growth by controlling the activity of YAP and TAZ (refs 11-13). YAP and TAZ are closely related transcriptional co-activators that promote the expression of pro-proliferative and anti-apoptotic genes. Upstream of YAP and TAZ are the Hippo kinases MST1/2 and LATS1/2, which negatively regulate YAP and TAZ and cause their exclusion from the nucleus. Loss of Hippo signalling leads to unrestricted proliferation in flies and mammals, and has been linked to a variety of developmental abnormalities and cancers ${ }^{14,15}$. NF2 can bind and recruit LATS to the plasma membrane, where it is activated by MST kinases ${ }^{16}$. NF2 has also been shown to bind other Hippo pathway components ${ }^{8}$. NF2 is one of many regulators of the Hippo pathway ${ }^{17}$ : Cell adhesion, cell polarity, mechanical forces and the cytoskeleton have all been shown to regulate YAP localization in tissue culture ${ }^{18}$, suggesting that as tissues grow and develop, feedback may occur from resultant changes in the environment.

Here we uncover an unsuspected role for NF2 and the Hippo pathway in kidney branching morphogenesis. We find that $N f 2$ conditional mutants have severe renal hypodysplasia due to defective branching morphogenesis. Nf2 kidney hypodysplasia can be rescued by reducing Yap and Taz dosage, and phenocopied by YAP overexpression, suggesting that NF2 restricts YAP/TAZ activity to promote branching morphogenesis. Lats $1 / 2$ deletion leads to kidney agenesis that can be rescued by reducing YAP/TAZ levels, suggesting that high YAP/TAZ activity inhibits branching. Importantly, loss of $\mathrm{Nf2}$, or overexpression of YAP inhibits RET signalling and expression of tip markers. Our mosaic analysis reveals that YAP overexpressing cells show cellautonomous loss of RET signalling and are excluded from UB tips. In contrast, we find that UB-specific deletion of Yap and Taz leads to cyst-like branching, associated with an expanded UB tip domain. Taken together, these data demonstrate an essential role for NF2 and the Hippo pathway in regulation of branching morphogenesis in the mammalian kidney.

\section{Results}

Nf2 deletion results in severe kidney defects. To investigate the role of NF2 during kidney development, we first stained developing kidneys with NF2 antibody, and found that NF2 protein uniformly localized along the apical membrane of the UB and nephrons throughout development (Fig. 1a and Supplementary Fig. 1a-h). NF2 is maintained in the adult kidney, although at a weaker level (Supplementary Fig. 1i). The apical staining is specific, as it is lost in Nf2 mutant tissue (Supplementary Fig. 1j-m).

To investigate the role of NF2 in UB branching, we deleted Nf2 within the UB lineage using Hoxb7:Cre (ref. 19). Hoxb7:Cre $e^{t g} /+$ $\mathrm{Nf} 2^{\text {flox/flox }}\left(\mathrm{Nf2} 2^{\mathrm{UB}-I_{-}}\right)$newborns (P0) were obtained at Mendelian ratios; however, only $6 \%$ of $N f 2^{U B-/-}$ pups survived to weaning suggesting early postnatal lethality. $N f 2^{U B-/-}$ newborns had severe kidney hypodysplasia (kidney area in $N f 2^{U B-/-}: \quad 1.1 \pm 0.7 \mathrm{~mm}^{2}, \quad n=14$; controls: $4.8 \pm 0.5 \mathrm{~mm}^{2}, n=18$, Fig. 1b-e). Histological examination of P0 kidneys revealed cortex-medulla disorganization and dilated tubules with limited number of glomeruli (Fig. 1f-h, Supplementary Fig. 1z,za). Staining for HNF $4 \alpha$ (proximal tubule marker), and CALBINDIN (collecting duct and distal tubule marker) revealed dilations were both autonomous and non-cellautonomous (Supplementary Fig. $1 \mathrm{n}-\mathrm{q}$ ). In rare cases where $\mathrm{Nf} 2^{U B-/-}$ animals survived to weaning, kidneys were smaller with tubule dilations and parenchyma destruction (Supplementary Fig. 1r,s).

NF2 is required for branching morphogenesis. To examine the role of NF2 in ureter branching in greater detail, we placed T-stage (2 UB tips) kidney rudiments dissected from wild-type and $N f 2^{U B-/-}$ E11.5 embryos in culture and counted the number of UB tips after $48 \mathrm{~h}$. Strikingly, deletion of $N f 2$ resulted in loss of ureter branching (number of UB tips: $2 \pm 0.4, n=21$ ) compared with controls ( $23.5 \pm 4.7, n=21$, Fig. 1i-k). Interestingly, while NF2 function is dispensable for the formation of the UB (marked by PAX2) and its first branching event (Fig. 1l,m), subsequent branching failed in the absence of $N f 2$ (Fig. $1 \mathrm{n}-\mathrm{q}$ and $2 a, b)$.

Despite the loss of branching, Nf2-depleted UBs continued to grow, as indicated by the wider epithelial tubules observed in $N f 2^{U B-/-}$ kidneys compared with controls (Fig. 1i,j and $2 c, d$ ). Quantification at E12.5 revealed a 2.2-fold increase in UB width and 1.85 -fold increase in cellularity in the UB compartment of $N f 2^{U B-/}-$ embryos compared with controls (Fig. 2e,f). At E12.5, the dense packing leads to the appearance of stratification; however, confocal z-stack imaging of PAX2/E-CADHERIN staining revealed that UB cells in Nf2 mutants contact both apical and basal surfaces, consistent with a pseudostratified structure (Supplementary Movie 1 (control) and Supplementary Movie $\left.2\left(N f 2^{U B-l-}\right)\right)$.

To ascertain if altered apoptosis could explain the morphological defects seen in $N f 2$ mutants, we examined CLEAVED 

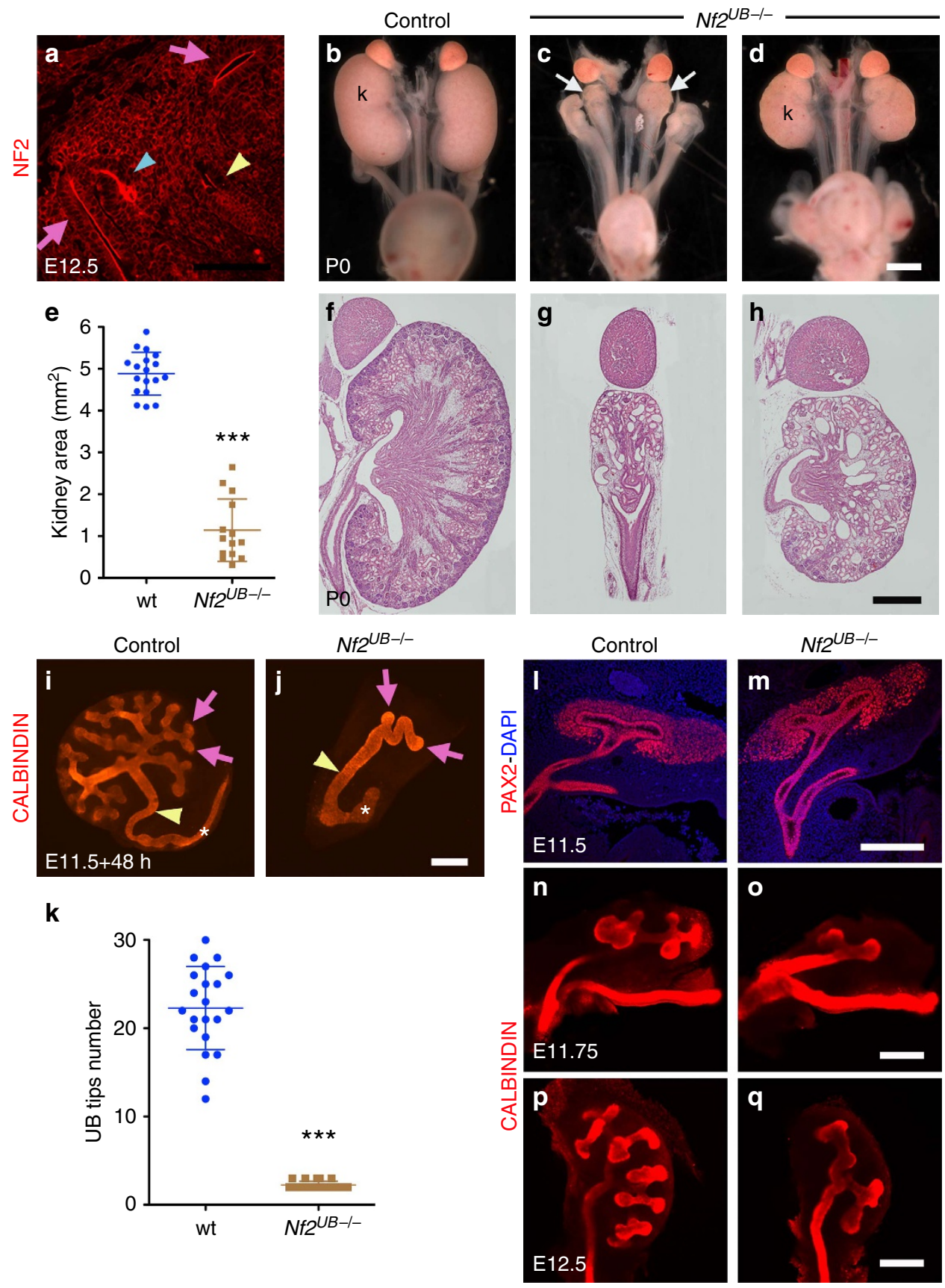

Figure 1 | Nf2 deletion leads to kidney defects due to defective branching morphogenesis. (a) NF2 protein is apically localized in all kidney epithelia: UB (pink arrows), collecting duct (yellow arrowhead) and early nephron (blue arrowhead). See Supplementary Fig. 1d for counterstaining with E-CADHERIN to mark the UB and early nephron. (b-d) Macroscopic view of the urogenital system from wild-type and Nf2UB-/- mutants at PO shows severe kidney hypoplasia (arrows) in Nf2 mutants. (e) Quantification revealed a 76\% decrease in kidney size in Nf2 mutants ( $n=14$ kidneys) compared with controls $(n=9)$. Error bars represent s.d., ${ }^{\star \star \star} P<0.0001$, Student's $t$-test. (f-h) Periodic acid-Schiff (PAS) staining of PO kidneys from wild type and Nf2 ${ }^{U B}-/-$. (i,j) Ex vivo analysis demonstrates loss of ureter branching in $N f 2^{U B-/-}$ compared with controls, visualized using anti-CALBINDIN antibody (pink arrows point to UB tips, yellow arrowheads to the ureter/collecting duct (CD) and asterisks mark the Wolffian duct). (k) Quantification of ureter branching capacity from ex vivo kidney explant experiments. For quantification, 21 kidneys explants were used in both genotypes. Error bars represent s.d.,

${ }^{\star \star \star} P<0.0001$, Student's $t$-test. The $y$ axis represents the number of ureteric tips after $48 \mathrm{~h}$ of culture. (I-q) Similar branching defects were observed in vivo using immunostaining on sections (PAX2 antibody, E11.5) and whole-mount immunostaining (CALBINDIN antibody, E11.75 and E12.5). Scale bars represent $100 \mu \mathrm{m}(\mathbf{a}), 1 \mathrm{~mm}(\mathbf{b}-\mathbf{d}), 0.5 \mathrm{~mm}(\mathbf{f}-\mathbf{h}), 250 \mu \mathrm{m}(\mathbf{i}, \mathbf{j})$ and $200 \mu \mathrm{m}(\mathbf{I}-\mathbf{q})$.

CASPASE 3 (CC3) staining in control and $N 2^{U B-/-}$ kidneys at E12.5. CC3-positive cells were rarely detectable in $N 2^{U B-I-}$ and control UB cells (marked by CALBINDIN) suggesting cell death is not the primary cause of defective branching (Supplementary Fig. 1t,u). Quantification revealed higher apoptotic rates in the cortex of $\mathrm{Nf}^{\mathrm{UB}-/-}$ compared with controls (Supplementary
Fig. 1v) consistent with known roles of branching in sustaining survival of the mesenchyme. Quantification of EdU incorporation at E11.5 showed no change in proliferation of UB cells (Supplementary Fig. 1w-y). We conclude that neither proliferation nor apoptosis are primarily responsible for $N f 2^{U B-/-}$ branching defects. 

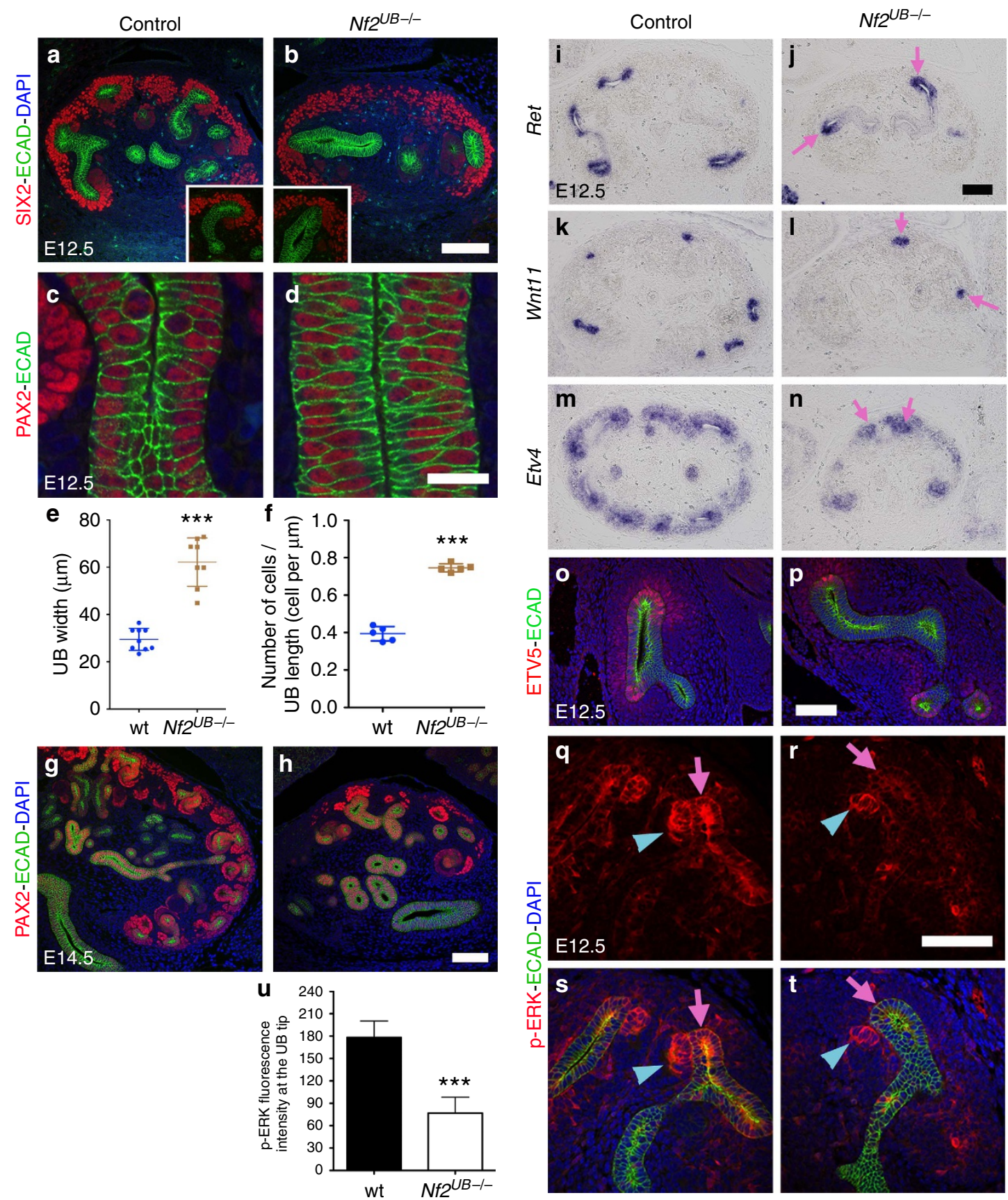

Figure 2 | Nf2 deletion leads to pseudostratification of the ureteric epithelium and reduced MAPK signalling (a,b) Loss of Nf2 leads to reduced UB branching, with sparse and reduced CM population at E12.5. (c,d) PAX2/E-CADHERIN staining reveals increased UB width and cellularity in Nf2 UB-/- mutants compared with controls at E12.5. (e,f) Quantification at E12.5 reveals a 2.2 and 1.85-fold increase in UB width and cellularity, respectively, in Nf2 ${ }^{U B}-/-$ compared with controls. (g,h) In Nf2 $\mathrm{UB}-/-$ kidneys, loss of CM cell population (PAX2) was apparent at E14.5. (i-p) UB tip-specific markers (in situ hybridizations: Ret, Wnt11, Etv4, immunostaining: ETV5) are expressed in the few UB tips that form in Nf2 mutants. (q-t) Phospho-ERK staining is greatly reduced in Nf2 UB-/UB tips compared with controls. Pink arrows point to UB tips, while blue arrowheads point to early nephron structures. (u) Quantification of $p$-ERK signal at the UB tips using five kidneys per genotype. Error bars represent s.d., ${ }^{\star \star \star} P<0.0001$, Student's $t$-test. Scale bars represent $100 \mu \mathrm{m}(\mathbf{a}, \mathbf{b}, \mathbf{g}$-t) and $20 \mu \mathrm{m}(\mathbf{c}, \mathbf{d})$.

Branching morphogenesis during kidney development involves signalling between the UB, metanephric mesenchyme and stromal compartments ${ }^{1,3}$. PBX1 staining revealed normal differentiation of the stromal compartment in $\mathrm{Nf2}$ mutants (Supplementary Fig. 2c,d). Staining with condensing mesenchyme (CM) markers (SALL1, SIX2 and PAX2) revealed a decreased CM population at E12.5 and E13.5, and a depletion of CM at E18.5 in $N f 2^{U B-/}$ - kidneys (Fig. 2a,b,g,h and Supplementary Fig. 2a-h). $\mathrm{PAX} 2$ staining on kidney explants confirmed the decreased CM population in Nf2 mutants compared with controls (Supplementary Fig. 2i,j). Moreover, we observed a difference in the morphology of the CM domain, as SIX2 cells appeared sparser in $N f 2^{U B}-/-$ kidneys compared with controls (compare SIX2 staining in Fig. 2a,b and Supplementary Fig. 2e,f).

NF2 is critical for RET-ERK signalling at UB tips. GDNF/RET signalling plays an essential role in UB branching ${ }^{5-7}$. GDNF 
binding to its receptors RET and GFR $\alpha 1$ induces both ERK/ MAPK and PI3K pathway activation. PI3K activation is critical for UB branching, and necessary for activation of downstream RET target genes ETV4 and ETV5 (ref. 20). Pharmacological inhibition of the ERK/MAPK pathway or loss of Mek1/2 also results in impaired branching but without affecting the expression of ETV4/5 (ref. 21). Thus both ERK/MAPK and PI3K signalling contribute to branching morphogenesis.

To test if the RET pathway is affected by Nf2 deletion, we performed in situ hybridization and immunostaining for Ret and its downstream targets. Although $N f 2^{U B-/}-$ kidneys had fewer UB tips compared with controls, Ret was expressed at levels comparable to controls at UB tips (Fig. 2i,j). We next examined RET signalling activity. No changes in Etv4, ETV5 and phosphoAKT were observed in $\mathrm{Nf2}^{\mathrm{UB}-/-}$ kidneys compared with controls (Fig. $2 \mathrm{~m}-\mathrm{p}$, Supplementary Fig. $2 \mathrm{~s}-\mathrm{v}$ ) suggesting that NF2 does not primarily affect PI3K activation. Interestingly, expression of tip marker genes (Wnt11, Crlf1, Sprouty1 and Dusp6) are seen at the few tips remaining in $\mathrm{Nf}^{\mathrm{UB}}{ }^{-/}-$kidneys (Fig. 2k,l, and Supplementary Fig. $2 \mathrm{~m}-\mathrm{r}$ ). We next examined ERK/MAPK pathway activation with antibodies to p-ERK (Fig. 2q-u). In controls, p-ERK staining was observed at the UB tips and in early nephrons. In $N f 2^{U B}-1-$ mutants, p-ERK staining persisted in early nephrons, but UB tip staining was greatly reduced suggesting that loss of $\mathrm{Nf2}$ may impair UB branching due to defective ERK/MAPK activation.

Yap/Taz haploinsufficiency rescues the $N f 2^{U B-/-}$ phenotype. Since NF2 is required for tight junction formation and cell polarity in the epidermis ${ }^{22}$, we examined $N f 2^{U B-1-}$ kidneys for CRUMBS3, ZO-1, $\beta$-CATENIN and LAMININ. All these markers revealed that UB cells depleted for $N f 2$ had normal apico-basal polarity at the time of defective branching (Supplementary Fig. 3a-f).

Nf2 deletion can inhibit the Hippo pathway, leading to reduced YAP/TAZ phosphorylation and increased nuclear YAP/TAZ (refs $16,23)$. We did not observe any changes in levels, phosphorylation or subcellular localization of YAP and TAZ in $N f 2^{U B-/-}$ compared with control kidneys at E12 (Supplementary Fig. 3gr). No significant changes were detected in western blot analysis using YAP, p-YAP, MST, p-MST, LATS and p-LATS antibodies in E13.5 $\mathrm{Nf2} \mathrm{UB-l}^{\mathrm{UB}}$ and control kidneys (Supplementary Fig. 3s). However, it is possible that we failed to detect subtle changes in YAP levels that nonetheless were functionally relevant. Therefore, we decided to genetically test whether excess YAP/TAZ activity in Nf2 mutants inhibits UB branching. We attempted to rescue the $N f 2^{U B-1-}$ phenotype by reducing YAP and TAZ levels, by

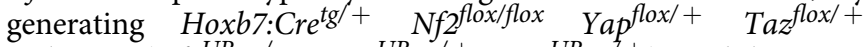
embryos (Nf2 $2^{U B-I_{-}} \mathrm{Yap}^{\mathrm{UB}-\mathrm{I}_{+}} \mathrm{Taz}^{\mathrm{UB}-\mathrm{Y}+}$ ). While E18.5 $N f 2^{U B-I-}$ mice had severe kidney hypodysplasia $\left(\mathrm{Nf2}^{\mathrm{UB}-{ }^{-}-}{ }^{-}\right.$: $1.1 \pm 0.7 \mathrm{~mm}^{2}$ ), removing one allele of each Yap and $T a z$ in $N f 2^{U B}-/-$ embryos remarkably restored kidney size ( $N f 2^{U B}-/-$ Yap ${ }^{U B-1+} \mathrm{Taz}^{\mathrm{UB}-1+}: 3.9 \pm 1.1 \mathrm{~mm}^{2}$ ) almost to wild-type size $\left(4.8 \pm 0.5 \mathrm{~mm}^{2}\right)$. Removal of either one copy of Yap $\left(\mathrm{Nf2} 2^{\mathrm{UB}-1-}\right.$ $\left.\mathrm{Yap} \mathrm{UB}^{\mathrm{UB}-1+}: 2.5 \pm 1.1 \mathrm{~mm}^{2}\right)$ or one copy of Taz $\left(\mathrm{Nf2} \mathrm{UB}^{\mathrm{UB}-1-}\right.$ $T a z^{U B-/+}: 2.2 \pm 1.1 \mathrm{~mm}^{2}$ ) partially rescued the phenotype (Fig. 3a-k)

To assess branching morphogenesis in greater detail, we dissected wild-type and $N f 2^{U B-1-} Y_{a p}{ }^{U-1+} T_{a z} U B-1+$ kidney rudiments at E11.5 and placed them in culture for $48 \mathrm{~h}$. Lowering YAP/TAZ dosage in Nf2 mutants largely rescued the branching capacity of kidney explants (Fig. 3l,m). Reducing YAP and TAZ also decreased the non-cell-autonomous tubule dilatation (Fig. 3n), and restored phospho-ERK expression (Fig. 3o-t). Remarkably, while most of Nf2 mutants died within
$48 \mathrm{~h}$ after birth, all $N f 2^{U B-/-} Y_{a p}{ }^{U B-I+} T a z^{U B-/+}$ mice lived to adulthood ( $n=5$ animals). These results suggest that the loss of branching morphogenesis in $N f 2$ mutants is due to excessive YAP/TAZ activity.

YAP expression reversibly inhibits branching. Our rescue experiments suggest that increased YAP/TAZ activities in $N f 2^{U B-/-}$ inhibit UB branching. To directly test this hypothesis, we overexpressed YAP in the UB lineage by generating Hoxb7: Cretg + Rosa26-lox-STOP-lox-rtta-IRES-EGFP Yap Tg animals (called $Y a p^{U B-O E}$ ). This approach allows constitutive expression of the wild type, HA-tagged form of YAP in the UB compartment after doxycycline treatment either by feeding pregnant dams with doxycycline food or by adding doxycycline to the kidney culture medium.

Initially, we overexpressed YAP in the UB lineage throughout kidney development by feeding pregnant dams with doxycycline $\left(0.625 \mathrm{~g} \mathrm{~kg}^{-1}\right)$ from E11 to E18.5. Yap ${ }^{U B-O E}$ animals developed severe renal hypodysplasia at E18.5 (Fig. 4a-d). To explore if YAP overexpression in the UB lineage affected branching, we cultured E11.5 Yap $\mathrm{YB}^{\mathrm{B}-O E}$ kidney rudiments (T-stage) in presence of doxycycline $\left(1,500 \mathrm{ng} \mathrm{ml}^{-1}\right)$, and counted the number of UB tips 24 and $48 \mathrm{~h}$ later. Interestingly, while control kidneys had $9 \pm 2.8$ and $22.5 \pm 1.3 \mathrm{UB}$ tips after 24 and $48 \mathrm{~h}$, respectively (Fig. $4 \mathrm{e}-\mathrm{g}, \mathrm{q}$ ), doxycycline-induced YAP overexpression in the UB led to a complete blockage of branching ( $2 \pm 0$ UB tips, Fig. $4 \mathrm{~h}-\mathrm{j}, \mathrm{q}$ and Supplementary Fig. 4a). Similarly, UB branching was blocked when doxycycline was added to the medium $24 \mathrm{~h}$ after dissection (Fig. 4k-m,q). Remarkably, when doxycycline was withdrawn from the medium, UB branching resumed (Fig. $4 \mathrm{n}-\mathrm{p}, \mathrm{q}$ ). Thus, YAP rapidly and effectively blocks branching in a reversible manner.

Constitutive YAP overexpression resulted in UB branching arrest (Fig. 4), but UBs overexpressing YAP continued to grow, as indicated by the wider epithelial tubules observed in Yap $U B-O E$ kidneys explants (Fig. 4i,j). Similarly, feeding pregnant dams with doxycycline diet from E11 to E13.5 was sufficient to activate YAP expression (Supplementary Fig. 5o,q), block UB branching, and led to increased UB width and epithelial pseudostratification in E13.5 Yap ${ }^{U B-O E}$ embryos (Fig. 5d,f). As with Nf2 mutants, proliferation in $Y a p^{U B-O E}$ mutants was unaffected at E13.5 (Supplementary Fig. 5e-g). Loss of Nf2 or YAP overexpression blocked branching and resulted in a pseudostratified epithelium. We speculate this is due to the continuation of proliferation in the absence of branching. Thus, increased YAP activity in the UB lineage leads to loss of branching.

YAP overexpressing cells are excluded from UB tips. YAP overexpression throughout the UB lineage inhibits branching morphogenesis and leads to kidney hypoplasia (Fig. 4). Interestingly, due to the occasional mosaic activity of the Hoxb7:Cre line, we observed that kidney hypoplasia correlated with the percentage of YAP overexpressing (YAP ${ }^{\mathrm{OE}}$ ) cells (Fig. $5 \mathrm{a}-\mathrm{g}$ ). We arbitrary divided the Yap $U B-\delta E$ animals in three categories (low, intermediate and high) based on the percentage of $\mathrm{YAPOE}$ cells in the ureteric epithelium. Kidneys with a low percentage of YAPOE cells $(<12 \%)$, were similar in size to controls, while those with a high percentage ( $>86 \%$ ) were severely reduced in size, likely as a consequence of branching arrest (Fig. 5g).

Additionally, we took advantage of the mosaicism to analyse the contribution of $\mathrm{YAP}^{\mathrm{OE}}$ cells to UB tips and trunks, compared with wild-type cells. We used an anti-HA antibody to identify cells that overexpressed transgenic YAP (HA-YAP), and PAX2 to label the entire ureteric tree. Remarkably, cells that overexpressed YAP rarely contributed to the UB tips (2.6\%), and their 

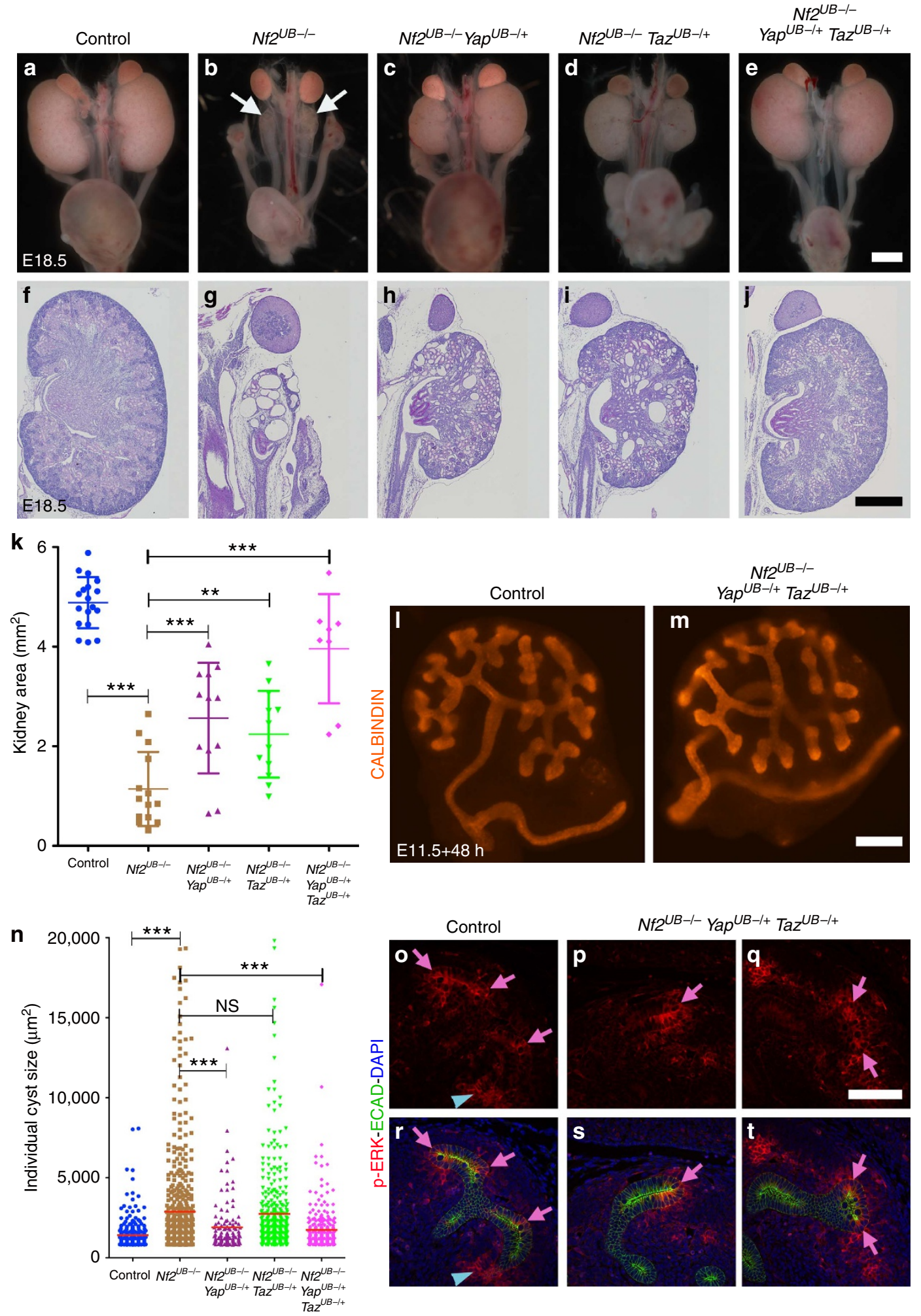

Figure 3 | Yap and Taz haploinsufficiency suppresses $\mathbf{N f 2} \mathbf{U}^{\mathbf{U B}}-\mathbf{/}-\mathbf{k i d n e y}$ hypodysplasia. Macroscopic (a-e) and periodic acid-Schiff (PAS) staining (f-j) of E18.5 kidneys reveals extent of rescue of the $N f 2 \mathrm{UB}-/-$ phenotype by Yap and/or Taz haploinsufficiency. (k) Quantification of kidney sizes in different

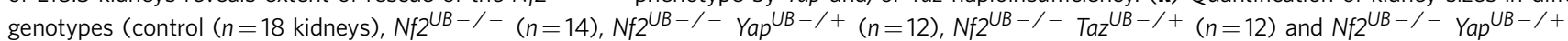

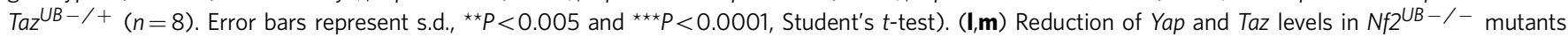
rescues branching in kidney explants. (n) Quantification of cyst sizes in different genotypes. (Control $(n=271 \mathrm{cysts}), N f 2^{U B-/-}(n=662), N f 2^{U B-/-}$ $\mathrm{Yap}^{U B-/+}(n=126), N f 2^{U B-/-T a z} \mathrm{UB-} /+(n=535)$ and $N f 2^{U B-/-} Y_{a p} U B-/+T a z U B-/+(n=316)$. Error bars represent s.d., NS, not significant, ${ }^{\star \star \star} P<0.0001$, Student's t-test). (o-t) Phospho-ERK expression is rescued in $N f 2^{U B-/}-Y_{a p} U B-/+T a z U B-/+$ UB tips to control levels. Pink arrows point to UB tips, while blue arrowheads point to early nephrons. Scale bars represent $1 \mathrm{~mm}(\mathbf{a}-\mathbf{e}), 0.5 \mathrm{~mm}(\mathbf{f}-\mathbf{j}), 200 \mu \mathrm{m}(\mathbf{I}, \mathbf{m})$ and $100 \mu \mathrm{m}(\mathbf{o}-\mathbf{t})$. 

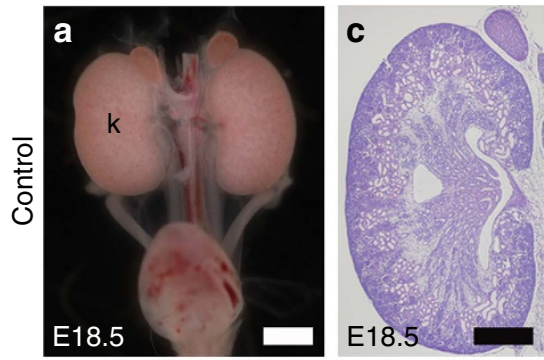

q
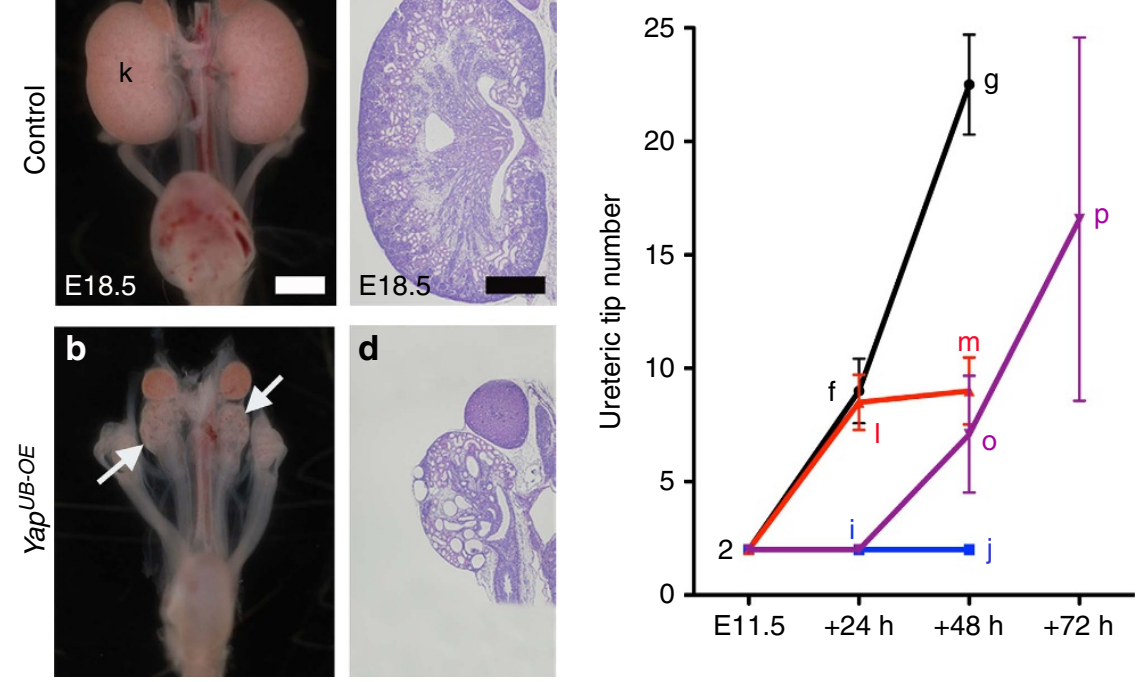

e

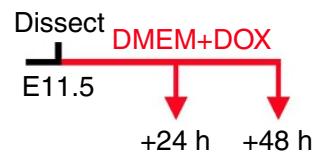

(f)

(g)

h

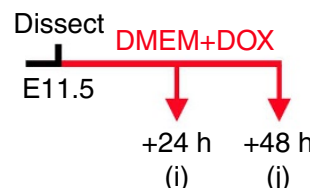

$\mathbf{k}$

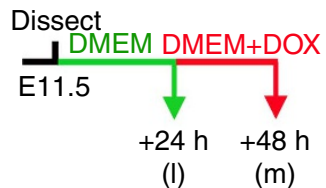

n

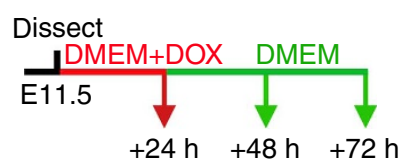

(i)

(o)
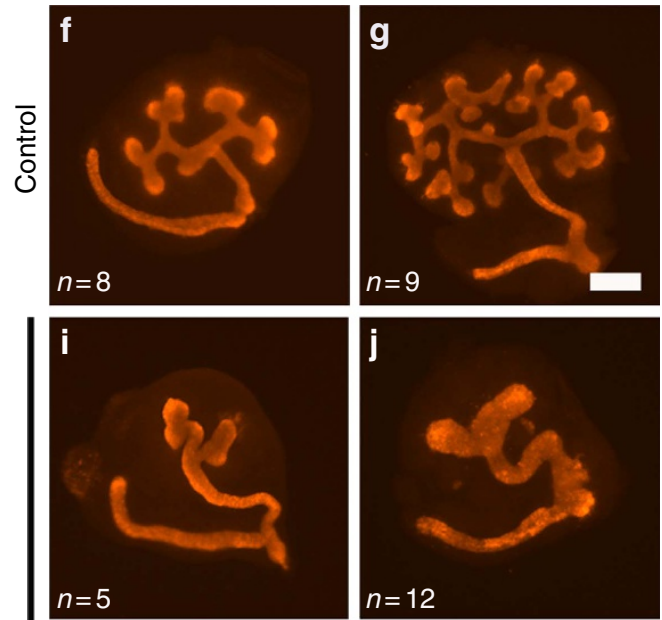

I
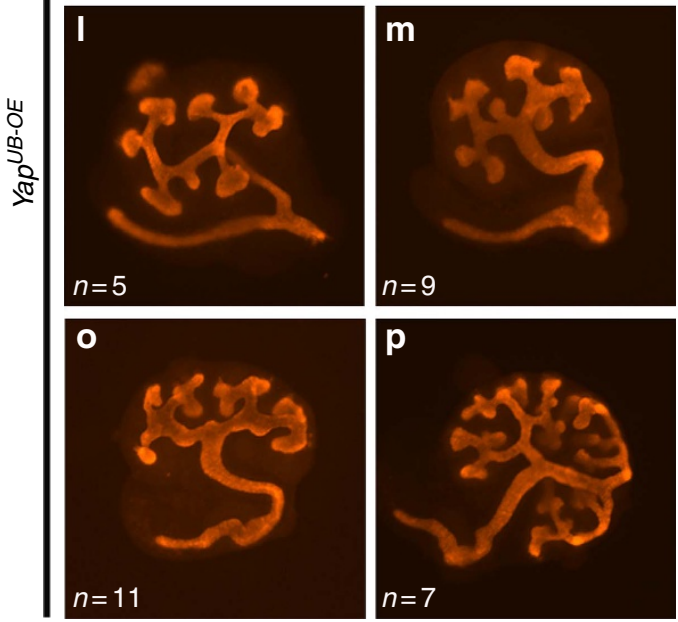

Figure 4 | YAP overexpression in the UB epithelium blocks branching. (a-d) Macroscopic view and periodic acid-Schiff (PAS) staining of YAP overexpressing and control kidneys at E18.5. Pregnant dams have been fed with doxycycline food $\left(0.645 \mathrm{~g} \mathrm{~kg}^{-1}\right)$ from E11 to E18.5. (e-p) E11.5 T-stage control kidney explants cultured ex vivo in presence of $1,500 \mathrm{ng} \mathrm{ml}^{-1}$ of doxycycline for 24 and $48 \mathrm{~h}$ shows the stereotypical branching morphogenesis pattern with an average of 22 ureteric tips in control kidneys after $48 \mathrm{~h}$ of cultures $(\mathbf{e}-\mathbf{g})$. (h-j) No branching is observed in Yap UB-OE kidneys as no new tip forms within 2 days in culture. ( $\mathbf{k}-\mathbf{m})$ After $24 \mathrm{~h}$ of culture in normal media, the addition of doxycycline and induction of YAP transgene expression results in a complete block of further branching. (n-p) Branching morphogenesis blockage requires continuous YAP overexpression, as withdrawal of doxycycline from the medium after $24 \mathrm{~h}$ releases the inhibition, and new tips are formed. (q) Quantification of UB tip number in ex vivo explants experiments. Panels $\mathbf{e}, \mathbf{h}, \mathbf{k}$ and $\mathbf{n}$ represent the experimental flow of the ex vivo kidney cultures. The number of explants analysed for each genotype is indicated in the lower left corner of each panel. Scale bars represent $1 \mathrm{~mm}(\mathbf{a}, \mathbf{b}), 0.5 \mathrm{~mm}(\mathbf{c}, \mathbf{d})$ and $250 \mu \mathrm{m}(\mathbf{f}-\mathbf{p})$. 

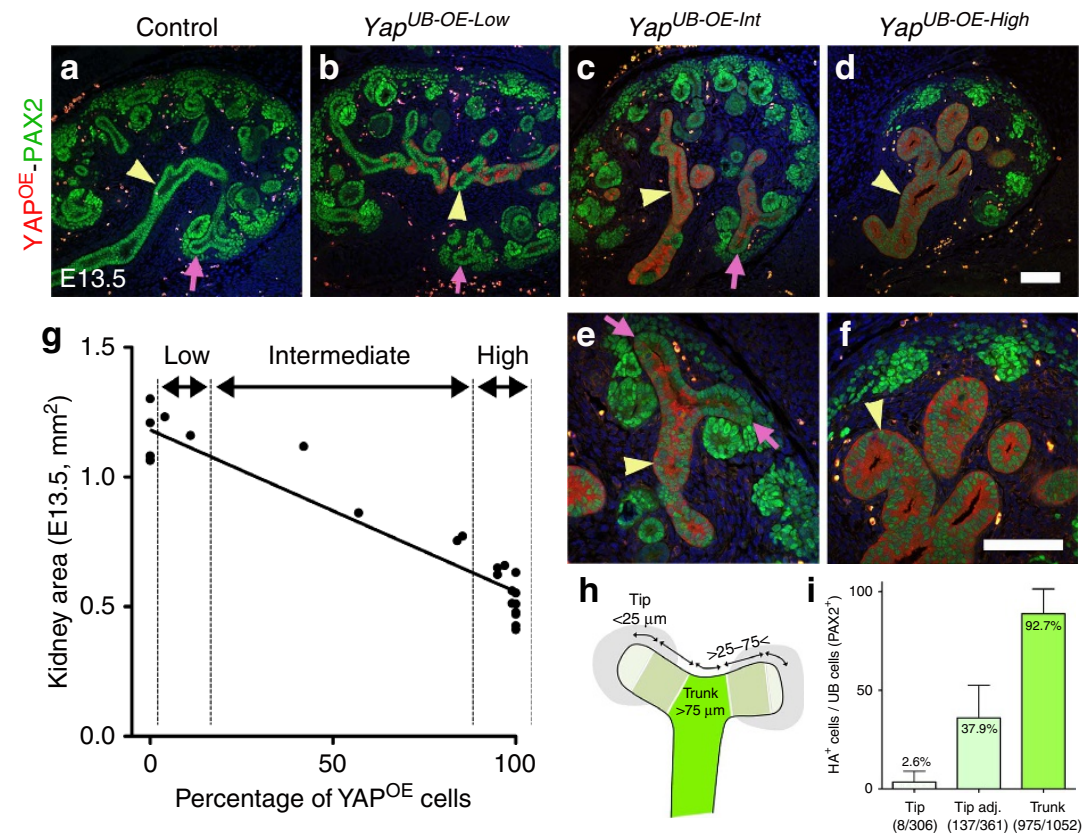

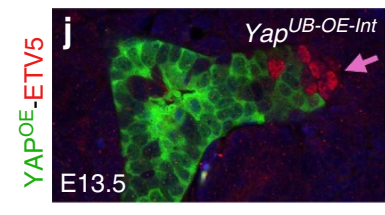

m

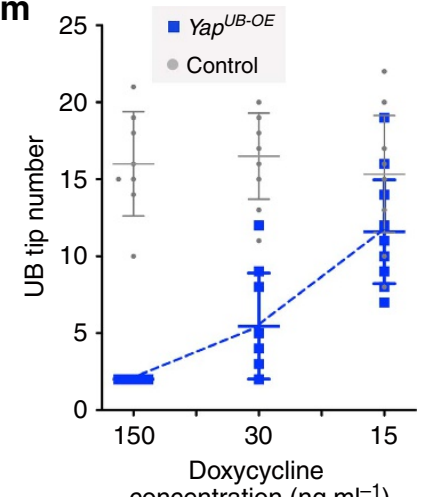

$\mathbf{r}$

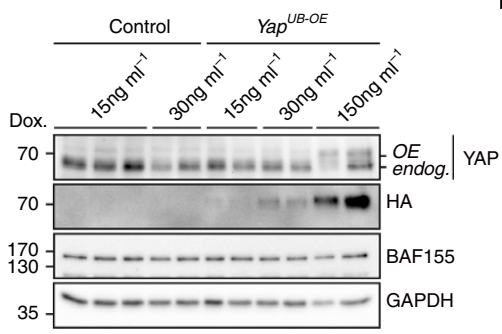

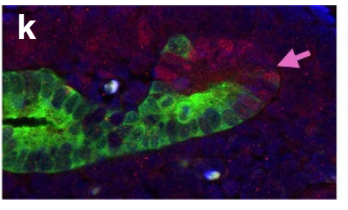

CALBINDIN
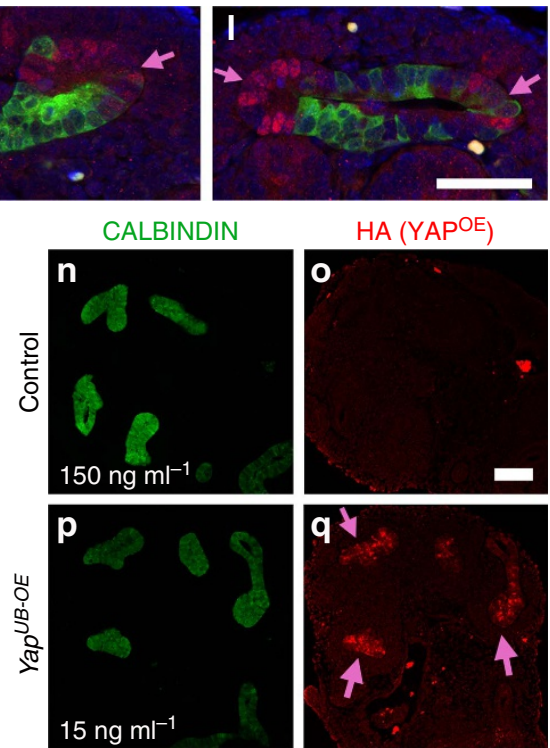

$\mathrm{HA}\left(\mathrm{YAP}^{\mathrm{OE}}\right)$

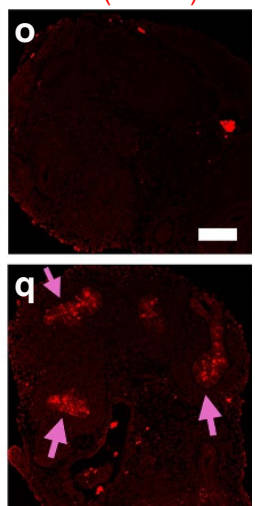

Figure 5 | Mosaic analysis reveals YAP overexpressing cells rarely contribute to UB tips. (a-I) Pregnant dams were fed with doxycycline food

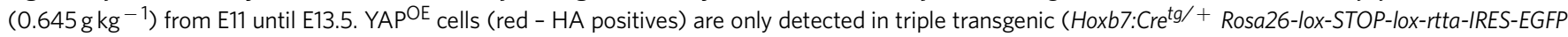
$\mathrm{Yap}^{T g}$ ) mice (b-f) and not in control animals (a). (e,f) Higher magnification views of $\mathbf{c}$ and $\mathbf{d}$. (g) Quantification reveals that the percentage of YAPOE cells in the UB epithelium correlates with kidney size. (h) Diagram of denomination of tip (within $25 \mu \mathrm{m}$ from the tip), tip adjacent (cells located $25-75 \mu \mathrm{m}$ away from the tip) and trunk (cells located $75 \mu \mathrm{m}$ and more from the tip) domains. (i) Quantification of the distribution of YAPOE cells in the UB compartments (tip, tip adjacent and trunk) in Yap ${ }^{U B-O E-I n t}$ mutants. Numbers in brackets represent the number of HA-positive cells and the total number of counted cells. (j-I) Immunostaining using anti-HA and ETV5 antibodies shows that the rare YAPOE cells present in the tip domain do not expressed the UB tip marker ETV5. Separate channels are shown in Supplementary Fig. $5 \mathrm{~h}-\mathrm{m}$. ( $\mathbf{m}$ ) Quantification of UB tip numbers of E11.5 control and Yap UB-OE kidneys, after $48 \mathrm{~h}$ in culture exposed to different concentrations of doxycycline $\left(15,30\right.$ and $150 \mathrm{ng} \mathrm{ml}^{-1}$ ). Quantification was made on 8, 10 and 12 control explants and 7, 10 and 12 Yap UB$O E$ explants at 150, 30 and $15 \mathrm{ng} \mathrm{ml}^{-1}$, respectively. (n-q) HA staining on kidney explant sections treated with $15 \mathrm{ng} \mathrm{ml}^{-1}$ of doxycycline (low enough to allow branching) reveals that cells with low YAP overexpression can contribute to UB tips. (r) Western blot analysis of kidney lysates confirms activation of YAP expression at different concentrations of doxycycline used in panels $\mathbf{m}-\mathbf{q}$. Scale bars represent $100 \mu \mathrm{m}(\mathbf{a}-\mathbf{f}, \mathbf{n}-\mathbf{q})$ and $50 \mu \mathrm{m}(\mathbf{j}-\mathbf{I})$. 
distribution increased away from the tips (37.9\% in tip adjacent domain), with nearly all trunk cells being HA positive $(92.7 \%$, Fig. 5c,e,i and Supplementary Fig. 5a,b). Even in low YAP overexpressing kidneys (Yap UB-OE-Low $)$, YAPOE cells were not randomly distributed, but exclusively found in the trunk (Fig. 5b, and Supplementary Fig. 5c,d). Importantly, in rare cases where HA cells did contribute to UB tips (2.6\%), expression of ETV5 was not detected (Fig. 5j-l and Supplementary Fig. 5h-m).

Next, we wondered if the tip exclusion behaviour of YAP $\mathrm{OE}$ cells is linked to the level of YAP overexpression. We first examined the impact of increasing doxycycline exposition (pregnant dams fed with $0.2,0.645$ (the same concentration used above) and $2 \mathrm{~g} \mathrm{~kg}^{-1}$ doxycycline food from E11 to E18.5) on kidney branching. Remarkably, all diets led to severe kidney hypoplasia (Supplementary Fig. 5n), likely due to the high level of YAP overexpression observed even at low dose (Supplementary Fig. 5o-r). Therefore, to better control the level of YAP overexpression, we examined the effect of varying the concentrations of doxycycline in cultured kidneys and analysed YAP induction after $24 \mathrm{~h}$ using western blot analysis. We also quantified UB branching after $48 \mathrm{~h}$ using CALBINDIN staining.
Decreasing the level of doxycycline to $15 \mathrm{ng} \mathrm{ml}^{-1}$ resulted in branching similar to controls (Fig. 5m), with HA-positive cells observed in the entire UB compartment (UB tip and trunk, as observed from kidney explant sections in Fig. $5 n-q)$. Thus cells with low levels of YAP overexpression (Fig. 5r) can contribute to active UB tips. We conclude that cells with high levels of YAP activity have a cell-autonomous defect in their ability to contribute to the UB tip domain and RET signalling.

LATS is required for branching via inhibition of YAP and TAZ. To test whether the core Hippo kinases LATS1 and LATS2 are essential for branching morphogenesis, we examined the consequence of removing Lats1 and Lats2 from the UB lineage

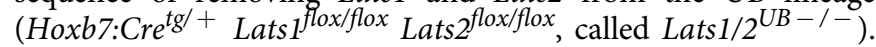
Normal kidney development was observed even in the absence of three out of four Lats1 and Lats2 alleles, regardless of allelic combinations (Supplementary Fig. 6a-c). However, double Lats1 and Lats 2 conditional knock-out embryos showed bilateral kidney agenesis at P0 (no kidneys observed in all four Lats $1 / 2 \mathrm{UB}-/-$ embryos, Fig. 6a,b). Earlier examination revealed that the UB
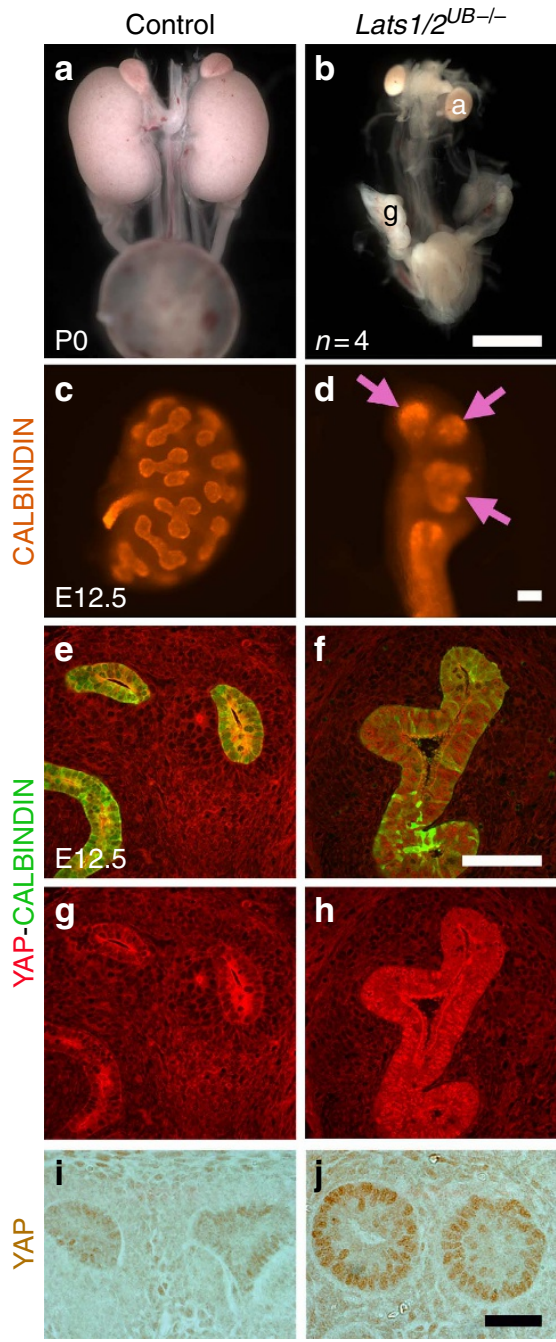

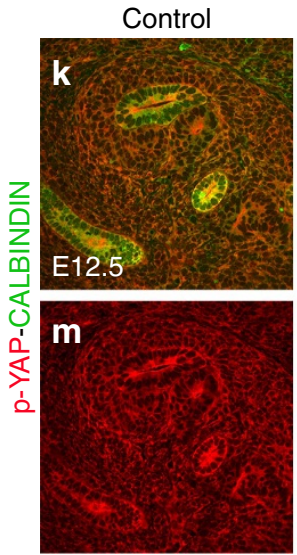

Lats $1 / 2^{U B-1-}$
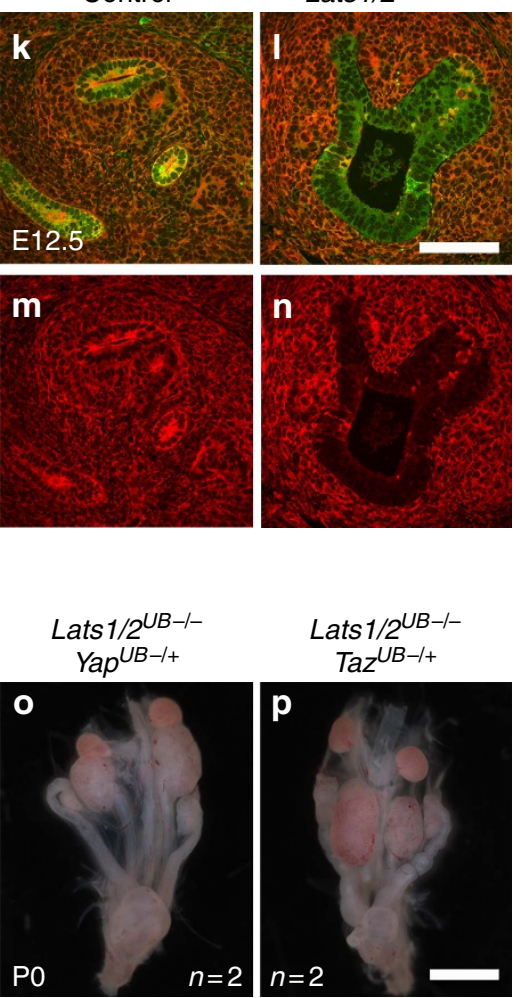

Figure 6 | The Hippo kinases LATS1 and LATS2 control branching through YAP/TAZ. (a,b) Macroscopic views of the urogenital system from controls and Lats $1 / 2^{U B-/-}$ mutants at PO. Note the bilateral kidney agenesis in double Lats $1 / 2^{U B-/-}$ embryos. a, adrenal; g, gonads. (c,d) Branching morphogenesis at E12.5 is severely reduced in Lats1/2 $2^{U-/-}$ embryos compared with controls as seen with whole-mount CALBINDIN immunostaining. Pink arrows point to the UB tips. (e-n) Lats1/2 deletion causes increased nuclear YAP (e-j), and decreased phospho-YAP (k-n) levels in the UB, marked by CALBINDIN. (o,p) Yap and Taz heterozygosity rescues the Lats $1 / 2 \cup B-/-$ kidney agenesis phenotype as kidneys form in $L a t s 1 / 2 \cup B-/-Y a p U B-/+$ and Lats $1 / 2^{U B-/-T a z} \cup B-/+$ embryos. Scale bars represent $1 \mathrm{~mm}(\mathbf{a}, \mathbf{b}, \mathbf{o}, \mathbf{p}), 100 \mu \mathrm{m}(\mathbf{c}-\mathbf{h}, \mathbf{k}-\mathbf{n})$ and $50 \mu \mathrm{m}(\mathbf{i}, \mathbf{j})$. 
formed, but subsequent branching morphogenesis was severely disrupted as seen by the reduced number of UB tips at E12.5 (control: $15 \pm 3.5$; Lats $1 / 2^{U B-/-}: 2 \pm 0.8$, Fig. $6 c, d$ ). Immunostaining of Lats $1 / 2^{U B-/}-$ kidneys at E12.5 showed increased nuclear YAP protein levels accompanied by a loss of phosphoYAP in the UB compartment compared with controls (Fig. 6e-n).
Importantly, introducing Yap or Taz haploinsufficiency into Lats1/2 mutants rescued kidney agenesis observed at birth (Lats1/ $2^{U B-I-Y a p^{U B-I+}}=4$ kidneys in 2 embryos, Lats $1 / 2^{U B-1-}$ $Y a p^{U B-1+}=4$ kidneys in 2 embryos, Fig. 6o,p). Interestingly, Lats1/2 deletion resulted in increased CRUMBS3 and E-CADHERIN apical staining, and abnormal localization of
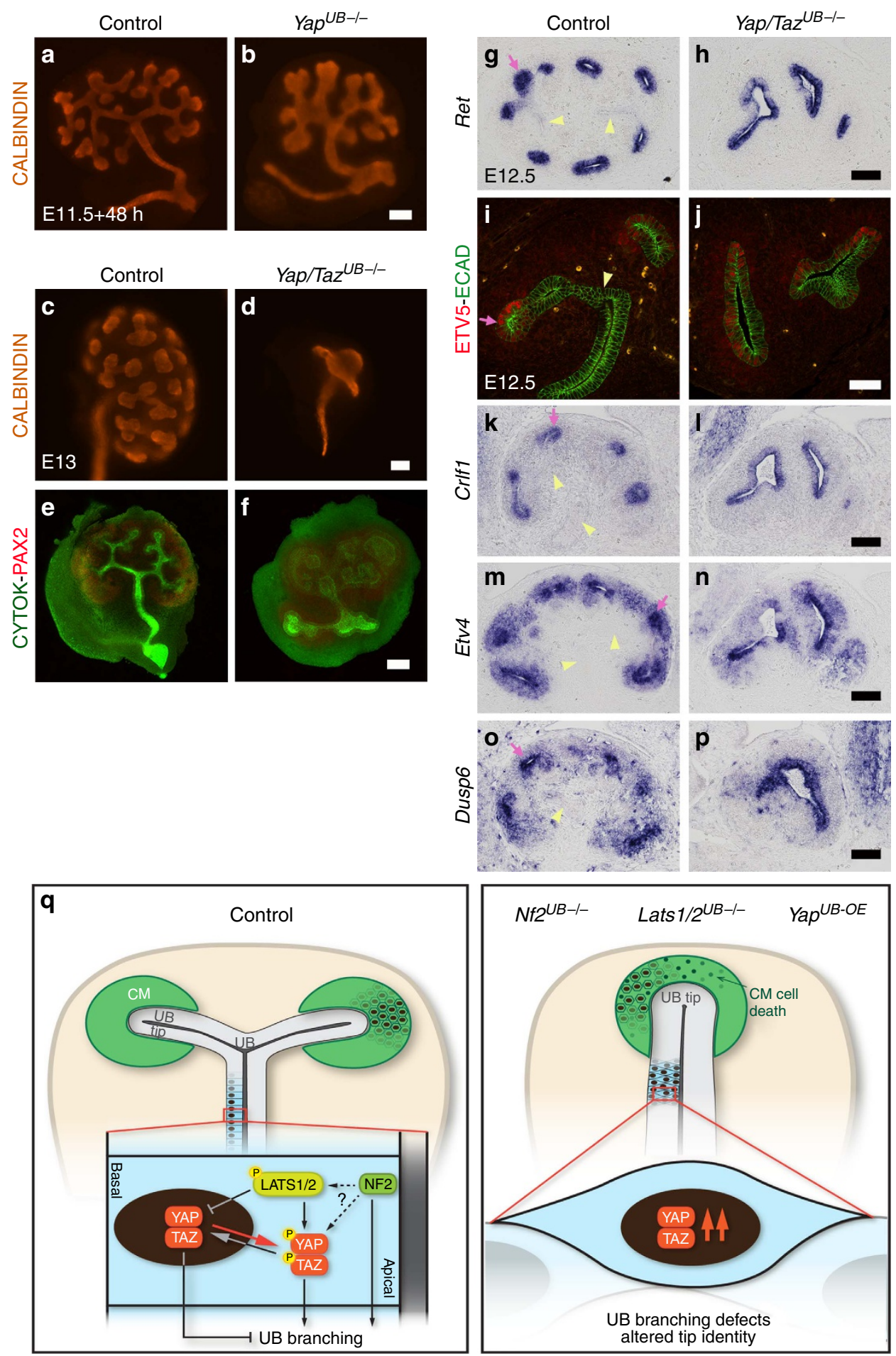

Figure 7 I YAP and TAZ control UB tip fate and structure. (a,b) Kidney explants of Yap UB-/- mutants show reduced branching with cyst-like morphology after $48 \mathrm{~h}$. (c-f) Whole-mount immunostaining reveals abnormal UB branching of Yap/Taz ${ }^{\mathrm{U}-1 /-}$ embryos compared with controls in vivo $(E 13, \mathbf{c}, \mathbf{d})$ and ex vivo (kidney culture, e, f). (g-p) In E12.5 controls, Ret, ETV5, Crlf1, Etv4 and Dusp6 are expressed in UB tips (pink arrows) but not in trunk segments (yellow arrowheads). In Yap/Taz UB-/- mutants, the entire UB epithelium expresses these genes, suggesting a shift towards tip identity. (q) Model figure: see discussion for description. Scale bars represent $100 \mu \mathrm{m}$. 
E-CADHERIN to the basal membrane (Supplementary Fig. 6d-i). Thus, the core Hippo kinases LATS1/2 are essential in the UB lineage to maintain cell polarity, restrict YAP/TAZ activity and to allow branching morphogenesis.

Yap/Taz deletion expands UB tip leading to dilated branching. Our data clearly show that high YAP/TAZ activity is incompatible with UB branching morphogenesis. To determine if Yap and $\mathrm{Taz}$ expression is essential for branching morphogenesis, we examined Yap and Taz mutants using the Hoxb7:Cre. Dilated tip structures were apparent in $Y a p^{U B-/-}$ kidney explants (Fig. 7a,b), while UB depleted of Taz branched normally (data not shown). Double Yap/Taz ${ }^{U B-/-}$ kidneys were hypoplastic with decreased proliferation (Supplementary Fig. $7 \mathrm{a}-\mathrm{c}$ ) and severe disruptions in branching morphogenesis, resulting in cyst-like branching (Fig. 7c-f). In controls, expression of Ret and its downstream targets ETV5, Crlf1, Etv4 and Dusp6 were restricted to the UB tips, whereas in Yap/Taz mutants, their expression domains expanded (Fig. $7 \mathrm{~g}-\mathrm{p}$ ). This suggests that in the absence of Yap and Taz, UB tip fate expands. Thus Yap/Taz deletion from the UB epithelium leads to the opposite effect from loss of $N f 2$ and excess YAP, namely expanded expression of RET pathway/ tip genes and cyst-like branching, that may represent unrestricted outward expansion of a tip fate.

\section{Discussion}

We have uncovered an unexpected role for NF2 and the Hippo pathway in regulating branching morphogenesis in the mammalian kidney. We found that NF2 and LATS1/2 are required in the UB lineage to restrain YAP/TAZ activities, and showed that YAP overexpression is sufficient to block branching morphogenesis. In addition, reducing YAP and TAZ levels can rescue branching defects of both Lats1/2 and Nf2 mutants. This suggests that increased YAP/TAZ activity is the primary cause of defective branching on loss of $N f 2$ and Lats1/2.

Using mosaic analysis, we also found that cells overexpressing YAP have a cell-autonomous defect in their ability to contribute to the UB tip domain. Thus, cells with heightened YAP levels, similar to Ret $^{-/-}$and $E t v 5^{-/-}$cells $^{3,24}$, are excluded from tips. Strikingly, rare $\mathrm{YAP}^{\mathrm{OE}}$ cells found at tips failed to activate RET signalling in a cell-autonomous manner. In contrast, UB depleted for Yap and Taz showed expansion of the tip domain. Taken together, these data suggest YAP/TAZ inhibits tip fate and signalling.

We cannot distinguish if YAP directly represses RET signalling or represses tip fate, thereby affecting RET signalling. Either scenario might allow a splitting of the tip, allowing new branch formation. How might this occur? One attractive scenario for the action of YAP/TAZ in symmetry breaking is that crowding that occurs at tips ${ }^{25}$, impacts the levels of nuclear YAP/TAZ and thereby RET signalling. This would split the tip domain, leading to branch formation. The positive feedback of RET signalling to increased Ret transcription would then promote further growth of each tip, until a critical point of cell crowding and tension occurs at a new ampulla, which would then again regulate nuclear YAP, causing splitting of the ampulla and branch formation.

Tip cells produce secreted paracrine signals that maintain and pattern both the stromal and mesenchymal compartments. Interestingly, $N f 2^{U B-/-}$ and $Y a p^{U B-O E}$ kidneys showed rapid depletion of the CM (as early as E13.5) suggesting that increased YAP activity at the tips is detrimental to the expression of an unidentified signal that maintains the CM. We noted cell death in the $N f 2^{U B-/}-$ outer cortex, likely due to the fact that CM cells receive reduced inductive signals from the smaller number of UB branches that formed.
Nf2 deletion leads to both cell-autonomous and non-cellautonomous tubule dilations at $\mathrm{P} 0$. The non-cell-autonomous effects may be due to secondary defects subsequent to loss of renal structure. Interestingly, lowering YAP and TAZ levels in the UB compartment of $N f 2$ mutants significantly reduced tubule dilatations suggesting that when NF2 inhibits YAP and TAZ in the UB compartment to promote branching morphogenesis, it may also restore inductive signals to the adjacent CM for nephron formation. It is interesting to note that while Yap or Taz haploinsufficiency partially rescues the branching defect of $\mathrm{Nf2}$ mutants, Taz haploinsufficiency has only a limited ability to rescue tubule dilatations compared with Yap haploinsufficiency. This confirms that YAP and TAZ have redundant function in branching ${ }^{26}$, and suggests that YAP and TAZ have different functions in restricting tubule dilations.

If both NF2 and LATS1/2 inhibit YAP/TAZ activity, why does Lats1/2 UB-deletion give rise to stronger phenotype than $N f 2$ deletion? Phospho-YAP staining persists in $N f 2^{U B-}-{ }_{-}$, while it is absent in Lats1/2UB-I- kidneys, suggesting that in the absence of Nf2, LATS1 and LATS2 kinases are still active. Our study suggests that LATS1/2 kinases might also have other roles than YAP/TAZ phosphorylation. We found that Lats $1 / 2 U B-/-$ displayed obvious polarity defects, not observed in $N f 2^{U B-/-}$ or Yap ${ }^{U B-O E}$ (Supplementary Fig. 4b-g). The suppression of defects of both Lats1/2 and Nf2 mutants by reducing YAP/TAZ dosage is consistent with a linear NF2-LATS1/2-YAP/TAZ activity pathway, but does not exclude other models (Fig. 7q).

While our genetic analysis clearly demonstrates an essential role for tight regulation of YAP/TAZ activity during branch formation, we could not detect a clear difference in the localization of YAP in the tips compared with the trunk. We speculate that changes in $\mathrm{YAP} / \mathrm{TAZ}$ localization are transient and/or only occur at low levels. We note that changes in Yorkie localization are not detectable in Drosophila discs in normally proliferating cells, suggesting that very low levels of YAP, undetectable by staining, can still be potent in transcriptional regulation. In vivo reporters of YAP/TAZ activity that can be imaged at real-time in the mouse kidney will be needed to obtain a better resolution of changes that are occurring at tips during development.

In conclusion, we have shown that NF2, LATS1/2, YAP and TAZ have critical roles in branching morphogenesis in the mammalian kidney. We propose a model in which the environment at the tip alters YAP/TAZ activity, which in turn feeds back to inhibit tip fate/signalling, break symmetry at the ampulla and promote branch formation. Since branching morphogenesis is critical for the formation of many organs, we speculate that the Hippo pathway will also play critical roles in branch formation in other tissues.

\section{Methods \\ Mouse lines. Hoxb7:Cre ${ }^{\text {tg/ }}+, \mathrm{Taz}^{\text {flox }}$ and Yap ${ }^{\text {flox }}$, Lats $1^{\text {flox }}$, Lats $2^{\text {flox }}, \mathrm{Nf} 2^{\text {flox }}$ and $Y a p^{T g}$ mouse strains were generously provided and have been described else- where ${ }^{19,27-30}$. All mice were maintained in a mixed genetic background and both male and female mice were assessed in our study. The age of all animals is indicated in the figure legends. Husbandry and ethical handling of mice were conducted according to guidelines approved by the Canadian Council on Animal Care. Genotyping was done by PCR using genomic DNA prepared from mouse ear punches.}

Histological and immunological analyses. Embryonic samples from timed matings (day of vaginal plug $=\mathrm{E} 0.5$ ) were collected, fixed in $4 \%$ paraformadehyde overnight $(\mathrm{O} / \mathrm{N})$ at $4{ }^{\circ} \mathrm{C}$, serially dehydrated and then embedded in paraffin. Microtome sections of $7 \mu \mathrm{m}$ thickness were examined histologically by periodic acid-Schiff staining. For immunofluorescent analysis, paraffin sections were dewaxed and serially re-hydrated with ethanol. Antigen retrieval was performed for $20 \mathrm{~min}$ in boiling Antigen Unmasking Solution (H-3300, Vector). Sections were incubated for $1 \mathrm{~h}$ in blocking solution (3\% BSA, 10\% goat serum, $0.1 \%$ Tween 20 in 
PBS) at room temperature (RT). Blocking solution was replaced by a solution of primary antibodies diluted in 3\% BSA, 3\% goat serum and 0.1\% Tween 20 in PBS. The following primary antibodies were used in this study: CALBINDIN (PC253C, Calbiochem, 1:300), CITED1 (RB-9219-P0, Neomarkers, 1:200), CLEAVEDCASPASE 3 (\#9661, Cell Signaling Technology, 1:300), CYTOKERATIN (C2562, Sigma, 1:200), CRUMBS3 (HPA013835, Sigma Prestige Antibodies, 1:300), E-CADHERIN (610181, BD Transduction Laboratories, 1:300), E-CADHERIN (\#3195, Cell Signaling Technology, 1:300), ETV5 (13011-1-AP, Proteintech, 1:200), HNF4 $\alpha$ (Gift from Marco Pontoglio, 1/300), LAMININ (L9393, Sigma, 1:300), LTL (FL-1321, Vector Laboratories, 1:300), NCAM (C9672, Sigma, 1:300), NF2 (HPA003097, Sigma Prestige Antibodies, 1:300), PAX2 (PRB-276P, Covance, 1:300), Phospho-AKT (\#4060, Cell Signaling Technology, 1:50), Phospho-ERK (\#4370, Cell Signaling Technology, 1:100), Phospho-YAP (\#4911, Cell Signaling Technology, 1:150), SALL1 (AB31526, Abcam, 1:300), SIX2 (11562-1-AP, Proteintech, 1:300), SOX9 (AB5535, Chemicon, 1:300), YAP (sc-101199, Santa Cruz Biotechnology, 1:150), YAP (\#14074, Cell Signaling Technology, 1:150), YAP/TAZ (\#8418, Cell Signaling Technology, 1:150) and ZO-1 (\#339100, Invitrogen, 1:500). Relevant conjugated secondary antibodies (Jackson Laboratories) were used for primary antibody detection. Slides were mounted using Vectashield with or without DAPI (Vector Labs). Fluorescent images were taken with a Nikon C1 plus Digital Eclipse confocal microscope.

YAP overexpression in Yap UB mice. Pregnant mice were fed with a doxycycline diet (Teklad Custom Diet, TD.120769, $18 \%$ protein) containing $0.625 \mathrm{~g} \mathrm{~kg}^{-1}$ of doxycycline hyclate. Doxycycline hyclate contains $\sim 87 \%$ of doxycycline. This diet is designed to deliver a daily dose of $2-3 \mathrm{mg}$ of doxycycline based on consumption of $4-5 \mathrm{~g}$.

Quantification of YAPOE cells in mosaic kidneys. Quantification was done following immunostaining of E13.5 kidney paraffin section stained with antibodies against PAX2 and HA (11 867423 001, Roche, 1:300). Ureteric tree was divided in three segments: UB tip cells (within $25 \mu \mathrm{m}$ from the tip), the tip adjacent region (cells located 25-75 $\mu \mathrm{m}$ away from the tip) and the trunk (cells located $75 \mu \mathrm{m}$ and more away from the tip). The number of HA-positive cells in each segment was quantified and divided by the total number of ureteric epithelial cells using an antiPAX2 antibody. Numbers in brackets (Fig. 5i) represent the number of HApositive cells per total number of UB PAX2-positive cells).

Whole-mount immunofluorescence. Embryos were dissected at suitable time points and fixed in $4 \% \mathrm{PFA} / \mathrm{PBS}\left(\mathrm{pH} 7.4\right.$ ) at $4{ }^{\circ} \mathrm{C} \mathrm{O} / \mathrm{N}$ then rinsed with PBS at RT several times. Urinary tracts were dissected under the microscope, soaked in PBSBB ( $1 \%$ BSA, $0.2 \%$ skim milk, $0.3 \%$ Triton X-100 and $1 \times$ PBS) to block and incubated $\mathrm{O} / \mathrm{N}$ at $4{ }^{\circ} \mathrm{C}$ on a shaker. Tissues were incubated with primary antibody solution diluted in PBS-BB at $4{ }^{\circ} \mathrm{C} \mathrm{O} / \mathrm{N}$, then washed with $\mathrm{PBS}-\operatorname{Tr}(0.3 \%$ Triton $\mathrm{X}-100,1 \mathrm{x}$ PBS) twice at RT for $1-2 \mathrm{~h}$ and once at $4{ }^{\circ} \mathrm{C} \mathrm{O} / \mathrm{N}$ on a shaker. Tissues were incubated with secondary antibody diluted in PBS-BB O/N at $4{ }^{\circ} \mathrm{C}$, washed several times in PBS-Tr at RT and imaged in $1 \times$ PBS on a Nikon C1 confocal system with NIS Elements software (Nikon Instruments Inc., America).

Kidneys explants. Kidney rudiments were dissected from E11.5 mouse embryos and place on filters (Millipore, $0.5 \mathrm{~mm}$ pore size) in direct contact with DMEM medium supplemented with $10 \%$ heat-inactivated foetal bovine serum, $10 \%$ glutamine and $1 \%$ penicillin/streptomycin. After 24 or $48 \mathrm{~h}\left(37^{\circ} \mathrm{C}\right.$ in $\left.5 \% \mathrm{CO}_{2}\right)$, kidney rudiments were fixed in ice-cold methanol at $4{ }^{\circ} \mathrm{C}$ while still attached to their filters, washed in PBS and blocked for $1 \mathrm{~h}$ in $2 \% \mathrm{BSA} / \mathrm{PBS}$ at RT. Staining was performed using CALBINDIN antibody (PC253C, Calbiochem; 1:200 diluted in PBS, $0.1 \%$ BSA, $0.1 \%$ Triton) followed by detection with a Cy3-conjugated anti-rabbit antibody (1:150, Jackson Laboratories) and kidneys were examined using a fluorescence microscope.

To induce YAP overexpression in kidney explants, doxycycline (R\&D Systems, AF2028) was added to the culture medium at different concentrations $(1,500,150$, 30 and $15 \mathrm{ng} \mathrm{ml}^{-1}$ ) from day 1 in culture, unless stated otherwise.

EdU incorporation. EdU solution containing 5-ethynyl-2' -deoxyuridine $\left(10 \mathrm{mg} \mathrm{ml}^{-1}\right)$ was injected intraperitoneally in pregnant mice $\left(50 \mathrm{mg} \mathrm{EdU} \mathrm{kg}^{-1}\right.$ of mice) $10-15 \mathrm{~min}$ before embryonic dissection. The samples were prepared and sectioned as described above and analysed using the Click-iT EdU Alexa Fluor 488 Imaging Kit (Life Technologies)

In situ hybridization. Anti-sense RNA probes labelled with digoxigenin (DIG, Roche) were prepared. Embryos were fixed in $4 \%$ paraformaldehyde in PBS O/N at $4{ }^{\circ} \mathrm{C}$, paraffin embedded and cut at $7 \mu \mathrm{m}$ and transferred onto superfrost glass slides. Fixed sections were hybridized $\mathrm{O} / \mathrm{N}$ with antisense RNA probes at $65^{\circ} \mathrm{C}$ in hybridization buffer. After several washes, sections were incubated in blocking buffer for $4 \mathrm{~h}$, followed by incubation with anti-DIG antibody (Roche) in blocking buffer $\mathrm{O} / \mathrm{N}$ at $4{ }^{\circ} \mathrm{C}$. After several washes, the colorimetric reaction was carried out using BM Purple (Roche).
Western blot. Western blot analysis was performed on stage E13.5 Nf2 ${ }^{U B-1-}$ kidneys or cultured Yap ${ }^{U B-O E}$ kidneys following doxycycline induction. Unless stated otherwise each western blot lane represents one animal (two kidneys). Kidneys were mechanically homogenized and lysed in RIPA buffer supplemented with proteasome and phosphatase inhibitors. Western blot analysis was performed following standard protocols with the following primary antibodies: CALBINDIN (C9848, Sigma, 1:1,000), BAF155 (SC-9748, SantaCruz Biotechnology, 1:2,000), GAPDH (R9545, Sigma, 1:7,500), HA (11867423001, Roche, 1:1,000), LATS1 (\#3477, Cell Signaling Technology, 1:1,000), MST1 (\#3682, Cell Signaling Technology, 1:2,000), NF2 (HPA003097, Sigma Prestige Antibodies, 1:2,000), phoshoLATS1 S909 (\#9157, Cell Signaling Technology, 1:500), phospho-MST1/2 T183/ T180 (\#3681, Cell Signaling Technology, 1:2,000), phospho-YAP S127 (\#4911, Cell Signaling Technology, 1:2,000) and YAP (sc-101199, SantaCruz Biotechnology, 1:2,000). All uncropped western blots can be found in Supplementary Fig. 8.

Cyst size quantification. Kidney masks were manually outlined in Fiji (PMID:22743772). Cyst segmentation was performed using Trainable Weka Segmentation v2.3.0 plugin for Fiji. The following features were used for pixel classification: Gaussian blur, difference of gaussians, Hessian, Sobel filter, variance and membrane projections. Objects were split in classes using Random Forest classifier Classification was refined for each image to assure accurate segmentation. Measurements for individual cysts were further processed using MATLAB 2015b (Mathworks)

Statistical analyses. All data are shown as mean values with s.d. An unpaired two-tailed $t$-test was used to determine differences between two groups (for example, wild type versus mutant). All statistical analyses were conducted using GraphPad Prism 5.0a software (La Jolla, USA).

Data availability. The authors declare that all data supporting the findings of this study are available within the article and its Supplementary Information files or from the corresponding authors on reasonable request.

\section{References}

1. Costantini, F. \& Kopan, R. Patterning a complex organ: branching morphogenesis and nephron segmentation in kidney development. Dev. Cell 18, 698-712 (2010).

2. Short, K. M. et al. Global quantification of tissue dynamics in the developing mouse kidney. Dev. Cell 29, 188-202 (2014).

3. Costantini, F. Genetic controls and cellular behaviors in branching morphogenesis of the renal collecting system. Wiley Interdiscip. Rev. Dev. Biol. 1, 693-713 (2012).

4. Shakya, R., Watanabe, T. \& Costantini, F. The role of GDNF/Ret signaling in ureteric bud cell fate and branching morphogenesis. Dev. Cell 8, 65-74 (2005).

5. Moore, M. W. et al. Renal and neuronal abnormalities in mice lacking GDNF Nature 382, 76-79 (1996).

6. Schuchardt, A., D’Agati, V., Larsson-Blomberg, L., Costantini, F. \& Pachnis, V. Defects in the kidney and enteric nervous system of mice lacking the tyrosine kinase receptor Ret. Nature 367, 380-383 (1994).

7. Cacalano, G. et al. GFRalpha1 is an essential receptor component for GDNF in the developing nervous system and kidney. Neuron 21, 53-62 (1998).

8. Cooper, J. \& Giancotti, F. G. Molecular insights into NF2/Merlin tumor suppressor function. FEBS Lett. 588, 2743-2752 (2014).

9. Lin, A. L. \& Gutmann, D. H. Advances in the treatment of neurofibromatosisassociated tumours. Nat. Rev. Clin. Oncol. 10, 616-624 (2013).

10. Schroeder, R. D., Angelo, L. S. \& Kurzrock, R. NF2/merlin in hereditary neurofibromatosis 2 versus cancer: biologic mechanisms and clinical associations. Oncotarget 5, 67-77 (2014).

11. Staley, B. K. \& Irvine, K. D. Hippo signaling in Drosophila: recent advances and insights. Dev. Dyn. 241, 3-15 (2012).

12. Zhao, B., Lei, Q. Y. \& Guan, K. L. The Hippo-YAP pathway: new connections between regulation of organ size and cancer. Curr. Opin. Cell Biol. 20, 638-646 (2008).

13. Halder, G. \& Johnson, R. L. Hippo signaling: growth control and beyond. Development 138, 9-22 (2011).

14. Pan, D. The hippo signaling pathway in development and cancer. Dev. Cell 19, 491-505 (2010)

15. Harvey, K. \& Tapon, N. The Salvador-Warts-Hippo pathway - an emerging tumour-suppressor network. Nat. Rev. Cancer 7, 182-191 (2007).

16. Yin, F. et al. Spatial organization of Hippo signaling at the plasma membrane mediated by the tumor suppressor Merlin/NF2. Cell 154, 1342-1355 (2013).

17. Yu, F. X., Zhao, B. \& Guan, K. L. Hippo pathway in organ size control, tissue homeostasis, and cancer. Cell 163, 811-828 (2015).

18. Dupont, S. et al. Role of YAP/TAZ in mechanotransduction. Nature 474, 179-183 (2011).

19. Zhao, H. et al. Role of fibroblast growth factor receptors 1 and 2 in the ureteric bud. Dev. Biol. 276, 403-415 (2004). 
20. Lu, B. C. et al. Etv4 and Etv5 are required downstream of GDNF and Ret for kidney branching morphogenesis. Nat. Genet. 41, 1295-1302 (2009).

21. Ihermann-Hella, A. et al. Mitogen-activated protein kinase (MAPK) pathway regulates branching by remodeling epithelial cell adhesion. PLoS Genet. 10, e1004193 (2014).

22. Gladden, A. B., Hebert, A. M., Schneeberger, E. E. \& McClatchey, A. I. The NF2 tumor suppressor, Merlin, regulates epidermal development through the establishment of a junctional polarity complex. Dev. Cell 19, 727-739 (2010)

23. Lavado, A. et al. Tumor suppressor Nf2 limits expansion of the neural progenitor pool by inhibiting Yap/Taz transcriptional coactivators. Development 140, 3323-3334 (2013).

24. Kuure, S., Chi, X., Lu, B. \& Costantini, F. The transcription factors Etv4 and Etv5 mediate formation of the ureteric bud tip domain during kidney development. Development 137, 1975-1979 (2010).

25. Packard, A. et al. Luminal mitosis drives epithelial cell dispersal within the branching ureteric bud. Dev. Cell 27, 319-330 (2013).

26. Reginensi, A. et al. Yap and Taz are required for Ret-dependent urinary tract morphogenesis. Development 142, 2696-2703 (2015).

27. Reginensi, A. et al. Yap- and Cdc42-dependent nephrogenesis and morphogenesis during mouse kidney development. PLoS Genet. 9, e1003380 (2013).

28. Heallen, T. et al. Hippo pathway inhibits Wnt signaling to restrain cardiomyocyte proliferation and heart size. Science 332, 458-461 (2011).

29. Giovannini, M. et al. Conditional biallelic Nf2 mutation in the mouse promotes manifestations of human neurofibromatosis type 2. Genes Dev. 14, 1617-1630 (2000).

30. Gregorieff, A., Liu, Y., Inanlou, M. R., Khomchuk, Y. \& Wrana, J. L. Yapdependent reprogramming of $\operatorname{Lgr} 5(+)$ stem cells drives intestinal regeneration and cancer. Nature 526, 715-718 (2015).

\section{Acknowledgements}

We thank Carlton Bates and Marco Giovannini for kindly providing mouse strains (Hoxb7:Cre $e^{t g /+}$ and $\mathrm{Nf} 2^{f l o x}$ respectively). We thank Mikhail Bashkurov at the Network

Biology Collaborative Centre for tubule dilatation quantification. This work was supported by grants from the Canadian Institutes of Health Research (MOP 136924, 84468), March of Dimes (1-FY11-506) and US Department of Defense (DOD, W81XWH-15-1-0461) to H.M. H.M. is a Tier 1 Canada Research Chair in Coordinating Growth and Polarity. Work in J.L.W. support from CIHR Foundation Scheme Program and the Krembil Foundation.

\section{Author contributions}

A.R. designed and performed all experiments. L.E. performed western blot analysis and graphical illustrations. A.G. and J.L.W. provided the Yap flox,$T a z^{f l o x}$ and $Y a p^{T g}$ mouselines. R.L.J. provided the Lats1/2flox mice. A.R. and H.M. wrote the manuscript.

\section{Additional information}

Supplementary Information accompanies this paper at http://www.nature.com/ naturecommunications

Competing financial interests: The authors declare no conflict of interest.

Reprints and permission information is available online at http://npg.nature.com/ reprintsandpermissions/

How to cite this article: Reginensi, A. et al. A critical role for NF2 and the Hippo pathway in branching morphogenesis. Nat. Commun. 7:12309 doi: 10.1038/ ncomms12309 (2016)

\section{(c) (1)}

This work is licensed under a Creative Commons Attribution 4.0 International License. The images or other third party material in this article are included in the article's Creative Commons license, unless indicated otherwise in the credit line; if the material is not included under the Creative Commons license, users will need to obtain permission from the license holder to reproduce the material. To view a copy of this license, visit http://creativecommons.org/licenses/by/4.0/

(C) The Author(s) 2016 